\title{
Evaluation of approximate stochastic Hodgkin-Huxley models
}

\author{
Ian C. Bruce \\ Department of Electrical \& Computer Engineering, McMaster University \\ Hamilton, ON, Canada ibrucedieee.org
}

\begin{abstract}
Fox and colleagues [1], [2] derived an algorithm based on stochastic differential equations for approximating the kinetics of ion channel gating that is substantially simpler and faster than "exact" algorithms for simulating Markov process models of channel gating. However, Mino and colleagues [3] argued that the approximation may not be sufficiently accurate in describing the statistics of action potential generation. Bruce [4] subsequently showed that some of the inaccuracies described in [3] were due to implementation choices, but several important inaccuracies remained. The objective of this study was to develop a framework for analyzing the remaining inaccuracies and determining their origin. Simulations of a patch of membrane with voltage-gated sodium and potassium channels were performed using an exact algorithm for the kinetics of channel gating and the approximate algorithm of Fox. The Fox algorithm assumes that channel gating particle dynamics have a stochastic term that is uncorrelated, zeromean Gaussian noise, whereas the simulation results of this study demonstrate that in many cases the stochastic term in the Fox algorithm should be correlated and non-Gaussian noise with a non-zero mean. The results indicate that the source of these differences in noise statistics is that the Fox algorithm does not adequately describe the combined behavior of the multiple activation particles in each sodium and potassium channel (three and four, respectively).
\end{abstract}

\section{INTRODUCTION}

The stochastic nature of ion channel gating is known to be physiologically significant for patches of membrane with small numbers of ion channels [5] such as the nodes of Ranvier in auditory nerve fibers [6]-[13]. In addition, there is evidence that the physiological noise from ion channel gating is perceptually significant for cochlear implants users [14][17]. This motivates the development of accurate and computationally efficient stochastic neural models.

Mino et al. [3] compared four different algorithms for implementing Hodgkin-Huxley models [18] with stochastic sodium channels: Strassberg and DeFelice [19], Rubinstein [6], Chow \& White [20], and Fox [1], [2]. The first three algorithms utilize exact methods for describing channel kinetics with finite-state Markov process models. In contrast, the algorithm of Fox uses stochastic differential equations (SDEs) to approximate the Markov process models. In addition to being simpler, the approximate method of Fox is around 7 times faster than the Chow \& White algorithm, the fastest of the exact methods [3].

However, for simulations of a patch of membrane with 1,000 sodium channels, Mino et al. [3] reported that the approximate method of Fox produced quite different action potential (AP) statistics than the other methods. They consequently argued that, in spite of its computational advantage, the Fox algorithm may be too inaccurate in some circumstances to use reliably as an approximation to the exact methods. Further analysis by Bruce [4] showed that some of the inaccuracies described in [3] were due to the method used in that study to determine the number of open sodium channels in the Fox algorithm. Mino et al. [3] rounded down the number of open sodium channels to an integer value, whereas Bruce [4] showed that more accurate results are obtained if the number of open sodium channels is rounded to the nearest integer [4]. However, several important inaccuracies remained, which appear to result from incorrect relative noise levels in the Fox model [4]. In this paper, a framework is presented for analyzing the inaccuracies of the Fox algorithm and determining their origin.

\section{METHODS}

\section{A. Models}

The Hodgkin-Huxley model sodium channel has three independent activation particles $m$ and one inactivation particle $h$, while the model potassium channel has four independent activation particles $n$ [18]. The Markov kinetics for gating of sodium and potassium channels are given by

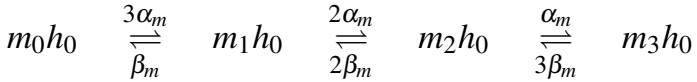

$$
\begin{aligned}
& \alpha_{h} \rrbracket \beta_{h} \quad \alpha_{h}\left\|\beta_{h} \quad \alpha_{h} \rrbracket \beta_{h} \quad \alpha_{h}\right\| \beta_{h}
\end{aligned}
$$

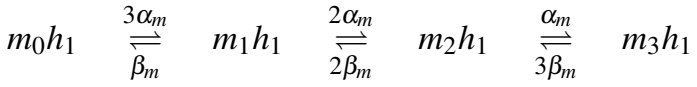

and

$$
n_{0} \underset{\beta_{n}}{\stackrel{4 \alpha_{n}}{\rightleftharpoons}} n_{1} \underset{2 \beta_{n}}{\stackrel{3 \alpha_{n}}{\rightleftharpoons}} n_{2} \underset{3 \beta_{n}}{\stackrel{2 \alpha_{n}}{\rightleftharpoons}} n_{3} \underset{4 \beta_{n}}{\stackrel{\alpha_{n}}{\rightleftharpoons}} n_{4}
$$

respectively, where $x_{i}$ indicates that $i$ particles of type $x$ are presently open in a particular channel.

If $N_{y}$ indicates the number of channels currently in state $y$, then the number of open sodium and potassium channels in a patch of membrane is given, respectively, by

$$
N_{\mathrm{Na}}(t)=N_{m 3 h 1}(t)
$$

and

$$
N_{\mathrm{K}}(t)=N_{n 4}(t) .
$$

There are several different numerical methods for simulating the Markov kinetics. [6] and [19] utilize methods that keep track of the gating particle states of every ionic channel, whereas the algorithm of [20] takes the more efficient 
approach of just keeping track of the number channels in each state.

Fox and colleagues [1], [2] proposed an alternative approach whereby the dynamics of the fraction of open gating particles $x$ is approximated by the SDE

$$
\frac{\mathrm{d} x(t)}{\mathrm{d} t}=\alpha_{x}(t)(1-x(t))-\beta_{x}(t) x(t)+\tilde{g}_{x}(t),
$$

where $x=m, h$, or $n$, the transition rates $\alpha_{x}(t)$ and $\beta_{x}(t)$ are instantaneous functions of the membrane potential $V(t)$, and the noise term $\tilde{g}_{x}(t)$ is Gaussian with moments

$$
\left\langle\tilde{g}_{x}(t)\right\rangle=0
$$

and

$$
\left\langle\tilde{g}_{x}(t) \tilde{g}_{x}\left(t^{\prime}\right)\right\rangle=\frac{2}{N_{\mathrm{X}}^{\max }} \frac{\alpha_{x}(t)(1-x(t)) \beta_{x}(t) x(t)}{2} \delta\left(t-t^{\prime}\right),
$$

where $\mathrm{X}=\mathrm{Na}$ for $x=m$ or $h$ and $\mathrm{X}=\mathrm{K}$ for $x=n$ and $N_{\mathrm{X}}^{\max }$ is the total number of ion channels of type $\mathrm{X}$.

Note that (5) is equivalent to the deterministic HodgkinHuxley ordinary differential equation for gating particle dynamics [18] but with the stochastic term $\tilde{g}_{x}(t)$ added.

Fox and colleagues [1], [2] showed that the noise term's $2^{\text {nd }}$ moment can be approximated by

$$
\left\langle\tilde{g}_{x}(t) \tilde{g}_{x}\left(t^{\prime}\right)\right\rangle=\frac{2}{N_{\mathrm{X}}^{\max }} \frac{\alpha_{x}(t) \beta_{x}(t)}{\alpha_{x}(t)+\beta_{x}(t)} \delta\left(t-t^{\prime}\right) .
$$

In the Fox algorithm, the number of open sodium and potassium channels is estimated to be

$$
N_{\mathrm{Na}}(t)=N_{\mathrm{Na}}^{\max } m^{3}(t) h(t)
$$

and

$$
N_{\mathrm{K}}(t)=N_{\mathrm{K}}^{\max } n^{4}(t),
$$

respectively, where $N_{\mathrm{Na}}^{\max }$ and $N_{\mathrm{K}}^{\max }$ are the total number of sodium and potassium channels, respectively, in the patch of membrane.

Numerical solution of (5) can be achieved by applying Euler's method to obtain the discrete-time difference equation [e.g., see Eq. 5 of [21]]

$$
x[k+1]=x[k]+\left\{\alpha_{x}[k](1-x[k])-\beta_{x}[k] x[k]\right\} \Delta t+\tilde{g}_{x}[k] \sqrt{\Delta t},
$$

where $\Delta t$ is the time step and $\tilde{g}_{x}[k]$ is a pseudorandom number with the statistics of $\tilde{g}_{x}(t)$.

\section{B. Framework for estimating Fox noise term from an "exact" algorithm}

Fox and colleagues [1], [2] did not derive an analytical expression for the error in their approximation. Presented here is an empirical method for estimating the required Fox noise term to match the channel gating statistics described by one of the "exact" models of the Markov kinetics [6], [19], [20].
From (1), the fraction of sodium channels with three open $m$ particles is given by

$$
\widehat{m}^{3}=\frac{N_{m 3 h 0}+N_{m 3 h 1}}{N_{\mathrm{Na}}^{\max }},
$$

and the fraction of sodium channels with an open $h$ particle is

$$
\hat{h}=\frac{N_{m 0 h 1}+N_{m 1 h 1}+N_{m 2 h 1}+N_{m 3 h 1}}{N_{\mathrm{Na}}^{\max }} .
$$

Likewise, (2) gives the fraction of potassium channels with four open $n$ particles as

$$
\hat{n}^{4}=\frac{N_{n 4}}{N_{\mathrm{K}}^{\max }} .
$$

Consequently, the Fox noise term that would be required to track the number of open channels at each time step $k$ computed from simulations using an exact method is

$$
\Delta \hat{g}_{x}[k]=\hat{x}[k+1]-\hat{x}[k]-\left\{\alpha_{x}[k](1-\hat{x}[k])-\beta_{x}[k] \hat{x}[k]\right\} \Delta t,
$$

where $\hat{x}=\hat{m}, \hat{h}$, or $\hat{n}$.

By comparing the statics of $\Delta \hat{g}_{m}[k], \Delta \hat{g}_{h}[k]$, and $\Delta \hat{g}_{n}[k]$ computed using (15) to the statistics of the analytical noise terms used in (11), i.e., $\tilde{g}_{m}[k] \sqrt{\Delta t}, \tilde{g}_{h}[k] \sqrt{\Delta t}$, and $\tilde{g}_{n}[k] \sqrt{\Delta t}$, a quantitative measure of the Fox approximation's accuracy is obtained.

\section{Simulations}

In order to understand how the channel gating statistics affect the auditory nerve fiber AP statistics investigated in [3] and [4], simulations are performed using the equations utilized in those studies for the sodium transition rates. In addition, simulations are performed using equations for the potassium transition rates that are also appropriate for the node of Ranvier in auditory nerve fibers [12].

Following [3] and [12], the activation and inactivation particle transition rates vary with the relative transmembrane potential $V$ according to

$$
\begin{gathered}
\alpha_{m}=\frac{1.872(V-25.41)}{1-\mathrm{e}^{(25.41-V) / 6.06}} \\
\beta_{m}=\frac{3.973(21.001-V)}{1-\mathrm{e}^{(V-21.001) / 9.41}} \\
\alpha_{h}=\frac{-0.549(27.74+V)}{1-\mathrm{e}^{(V+27.74) / 9.06}} \\
\beta_{h}=\frac{22.57}{1-\mathrm{e}^{(56.0-V) / 12.5}} \\
\alpha_{n}=\frac{0.129(V-35)}{1-\mathrm{e}^{(35-V) / 10}} \\
\beta_{n}=\frac{0.3236(35-V)}{1-\mathrm{e}^{(V-35) / 10}},
\end{gathered}
$$

where the rates have units of $\mathrm{ms}^{-1}$ and $V$ has units of $\mathrm{mV}$. 
Equation (8) indicates that the dominant factor in the Fox noise term statistics is the present value of the transition rates, which in turn depend only on the present value of the relative transmembrane potential-see (16)-(21). Therefore simulations were run in voltage clamp condition, i.e., at a constant relative transmembrane potential.

Voltage clamp simulation were performed with a sampling step $\Delta t$ of $1 \mu \mathrm{s}$ and a duration of $1 \mathrm{~s}$. The number of sodium channels $N_{\mathrm{Na}}^{\max }$ was varied from 100 to 20,000 , with the number of potassium channels $N_{\mathrm{K}}^{\max }$ always set to one third of the number of sodium channels (rounded to the nearest integer).

Results shown below were obtained with the Chow \& White algorithm [20]; some simulations were also run using the Rubinstein algorithm [6] and were found to give similar results.

\section{RESULTS}

Simulation results for $N_{\mathrm{Na}}^{\max }=1,000$ and $N_{\mathrm{K}}^{\max }=333$ are described here. First, an analysis of gating particle dynamics at $V=16 \mathrm{mV}$ is given. This relative transmembrane potential is of interest because that is approximately $2 / 3$ the threshold potential in the model of [3] and [4], and consequently the gating particle dynamics at this potential will have a substantial effect on the statistics of AP generation. Second, an analysis of the noise terms' standard deviations and means as a function of the membrane potential is described.

Figure 1 gives an analysis of the dynamics of sodium activation particles at $V=16 \mathrm{mV}$. The top two panels show that at this transmembrane potential the Fox noise term required to match the $m$-particle dynamics from the Chow \& White algorithm has small values near zero for most time steps but has infrequent large values in positive and negative pairs. These values are well outside the Gaussian distribution of values from the Fox algorithm. The autocorrelation function in the bottom right panel indicates that these large positive and negative values are correlated on a time scale of several microseconds, unlike the Fox noise term, which is uncorrelated. The example time series for $\hat{m}$ plotted in the bottom left panel shows that these correlated noise values correspond to brief openings or closings of small numbers of gating particles.

An analysis of the dynamics of sodium inactivation particles at $V=16 \mathrm{mV}$ is given in Fig. 2. The histogram of noise values shown in the top right panel indicates that at this transmembrane potential the Fox noise term required to match the $h$-particle dynamics from the Chow \& White algorithm has a distribution of finite values with a similar shape, mean and standard deviation to the Gaussian distribution of values from the Fox algorithm. The autocorrelation function in the bottom right panel indicates that the estimated noise term for sodium inactivation particles is uncorrelated like the Fox noise term.

Figure 3 gives an analysis of the dynamics of potassium activation particles at $V=16 \mathrm{mV}$. The top two panels show that at this transmembrane potential the Fox noise term required to match the $n$-particle dynamics from the Chow
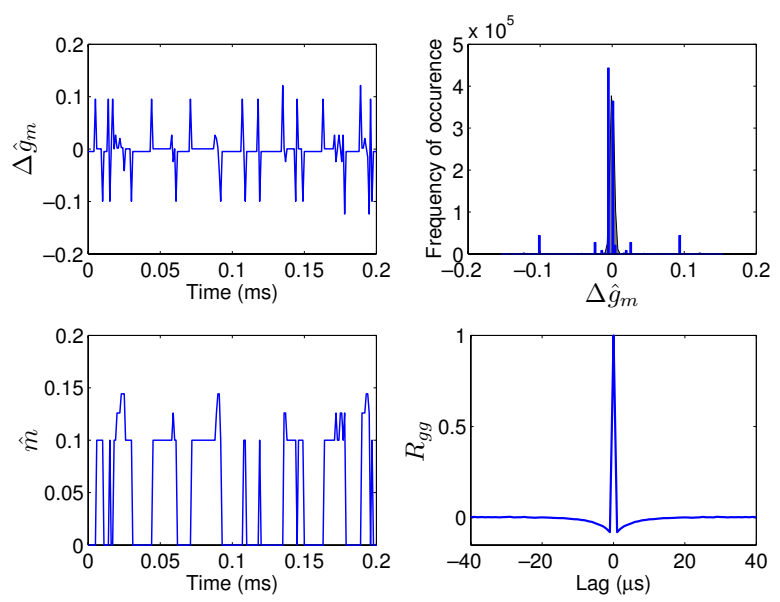

Fig. 1. Activation dynamics for 1,000 sodium channels: analysis of $m$ particle dynamics for relative transmembrane potential $V=16 \mathrm{mV}$. Top left panel: Example time series of $\Delta \hat{g}_{m}$. Top right panel: Histogram of values of $\Delta \hat{g}_{m}$. The theoretical distribution derived by Fox and colleagues [1], [2] is shown by the grey-filled Gaussian curve. Bottom left panel: Time series of $\hat{m}$ corresponding to top left panel. Bottom right panel: Autocorrelation function for $\Delta \hat{g}_{m}$.
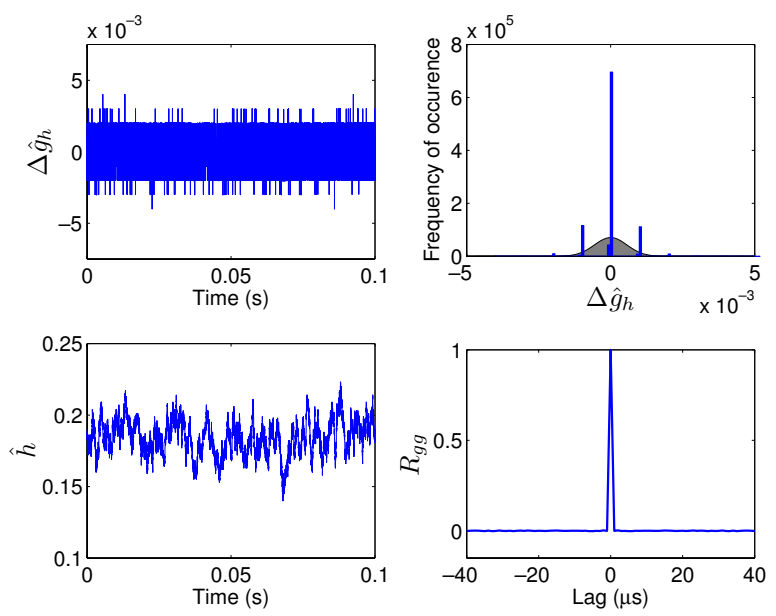

Fig. 2. Inactivation dynamics for 1,000 sodium channels: analysis of $h$ particle dynamics for relative transmembrane potential $V=16 \mathrm{mV}$. Top left panel: Example time series of $\Delta \hat{g}_{h}$. Top right panel: Histogram of values of $\Delta \hat{g}_{h}$. The theoretical distribution derived by Fox and colleagues [1], [2] is shown by the grey-filled Gaussian curve. Bottom left panel: Time series of $\hat{h}$ corresponding to top left panel. Bottom right panel: Autocorrelation function for $\Delta \hat{g}_{h}$.

\& White algorithm has very small values near zero for most time steps but has one pair of very infrequent large positive and negative values indicated by the arrows. As was the case for sodium activation particles, these values for potassium are well outside the Gaussian distribution of values from the Fox algorithm. The autocorrelation function in the bottom right panel indicates that these large positive and negative values are correlated on a time scale of tens of microseconds. The example time series for $\hat{n}$ plotted in the bottom left panel shows that these correlated noise values correspond to brief openings of one out of the 333 potassium channels.

Shown in Figs. 4, 5, and 6 are plots of the noise term standard deviation and mean as a function of $V$ for sodium 

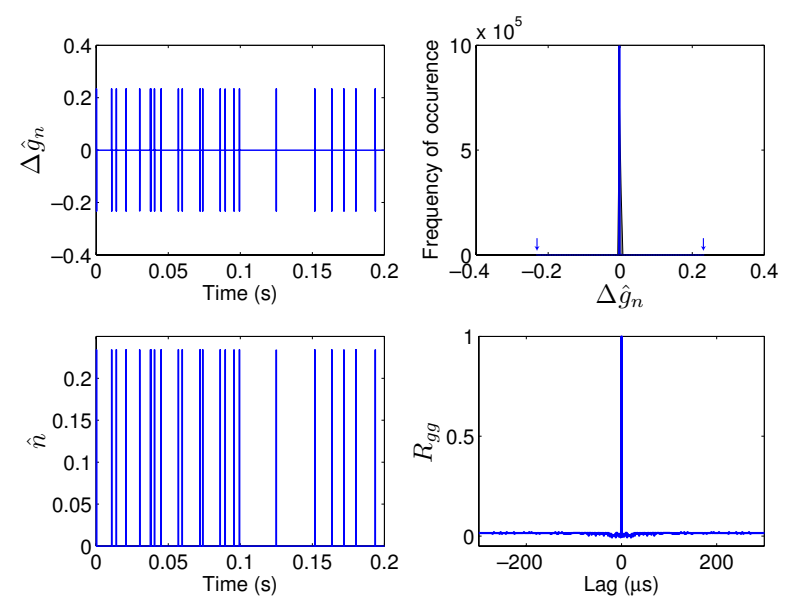

Fig. 3. Activation dynamics for 333 potassium channels: analysis of $n$ particle dynamics for relative transmembrane potential $V=16 \mathrm{mV}$. Top left panel: Example time series of $\Delta \hat{g}_{n}$. Top right panel: Histogram of values of $\Delta \hat{g}_{n}$. The theoretical distribution derived by Fox and colleagues [1], [2] is shown by the grey-filled Gaussian curve. Bottom left panel: Time series of $\hat{n}$ corresponding to top left panel. Bottom right panel: Autocorrelation function for $\Delta \hat{g}_{n}$.

activation, sodium inactivation, and potassium activation, respectively. The solid dark blue lines correspond to the standard deviation calculated for the noise term time series at the sampling step of $1 \mu$ s. The $h$-particle curve (which is mostly obscured by the green line) is close to the theoretical curve (black line) from the Fox algorithm (Fig. 5). However, the $m$-particle and $n$-particle curves (Figs. 4 and 6 ) are quite different from their corresponding theoretical curves (black lines); the peaks occur at a lower membrane potential than the theoretical curves, and they have substantially larger maxima.

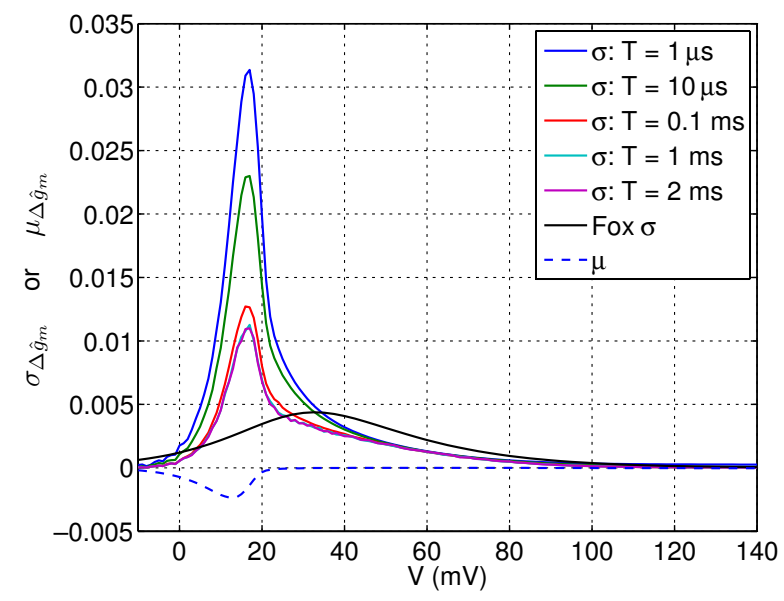

Fig. 4. Activation statistics for 1,000 sodium channels: standard deviation and mean of $\Delta \hat{g}_{m}$ as a function the relative transmembrane potential $V$. Standard deviations are calculated for values of $\Delta \hat{g}_{m}$ averaged over contiguous time windows of duration $T$, as indicated in the figure legend. The theoretical standard deviation versus membrane potential relationship given by (8) is shown for comparison.

The gating particles noise terms' means are shown by the dashed blue lines. Again, the $h$-particle curve (Fig. 5) matches the theoretical mean of zero from the Fox algorithm,

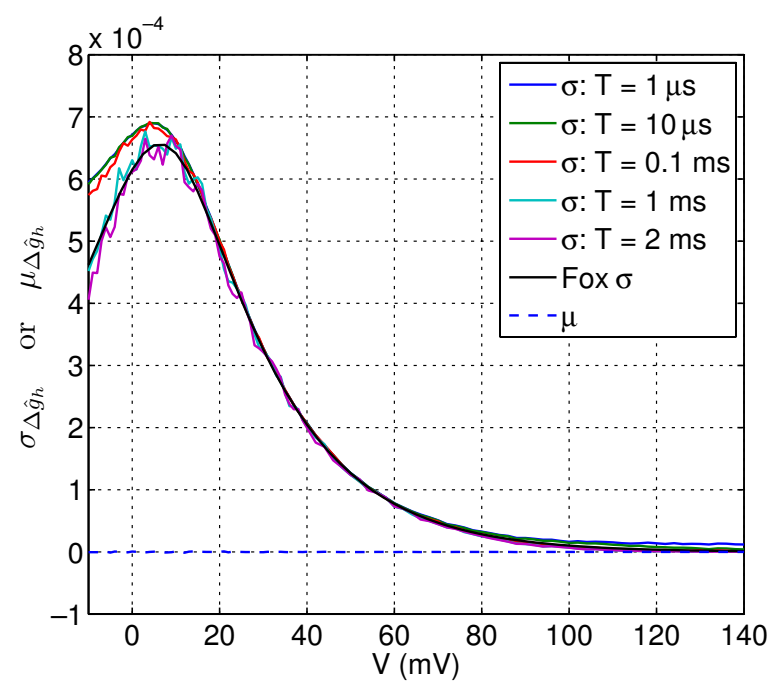

Fig. 5. Inactivation statistics for 1,000 sodium channels: standard deviation and mean of $\Delta \hat{g}_{h}$ as a function the relative transmembrane potential $V$. Standard deviations are calculated for values of $\Delta \hat{g}_{h}$ averaged over contiguous time windows of duration $T$, as indicated in the figure legend. The theoretical standard deviation versus membrane potential relationship given by (8) is shown for comparison.

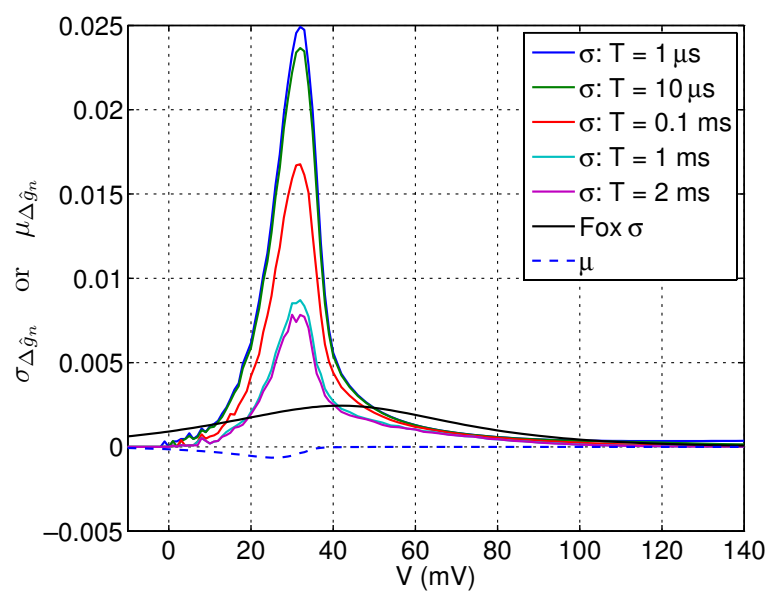

Fig. 6. Activation statistics for 333 potassium channels: standard deviation and mean of $\Delta \hat{g}_{n}$ as a function the relative transmembrane potential $V$. Standard deviations are calculated for values of $\Delta \hat{g}_{n}$ averaged over contiguous time windows of duration $T$, as indicated in the figure legend. The theoretical standard deviation versus membrane potential relationship given by (8) is shown for comparison.

whereas the $m$-particle and $n$-particle means (Figs. 4 and 6) have appreciably-large negative values in the range of the resting potential $(0 \mathrm{mV})$ to the AP threshold potential $(\sim 24 \mathrm{mV})$ for the model of [3] and [4].

Since the $m$-particle and $n$-particle noise term time series estimated from the Chow \& White algorithm both exhibit correlations over time and these noise terms have an accumulative effect on the $m$-particle and $n$-particle values according to (11), it is of interest to determine the effective standard deviations of the noise terms accumulated over different time scales. To do this, standard deviations are calculated for values of $\Delta \hat{g}_{m}, \Delta \hat{g}_{h}$, and $\Delta \hat{g}_{n}$ averaged over contiguous time windows of duration $T$. Included in Figs. 4, 5, and 
6 are standard deviation curves for different values of $T$, as indicated by the figure legends. The standard deviation curves all begin to reach their respective asymptotic curves at an integration period of $T=1 \mathrm{~ms}$, indicating that all the correlations in each noise term time series have been averaged out over this time period. The $h$-particle standard deviation curve asymptote matches the theoretical curve (Fig. 5); the $m$-particle and $n$-particle standard deviation curve asymptotes have smaller maxima than their respective curves without integration (i.e., $T=1 \mu \mathrm{s}$ ) but do not match their respective theoretical curves (Figs. 4 and 6).

The simulations results obtained for different numbers of sodium channels $N_{\mathrm{Na}}^{\max }$ and potassium channels $N_{\mathrm{K}}^{\max }$ were qualitatively similar to the results shown above. Just as the theoretical standard deviation scales with the reciprocal of the number of channels according to (7), the standard deviations estimated from the Chow \& White algorithm also scale with the reciprocal of the number of channels, such that the inaccuracies remain even for the largest numbers of channels investigated in this study.

From the results described above, it can be seen that the Fox algorithm provides a reasonable approximation of the single sodium channel inactivation particle $h$ but not of the three sodium or four potassium activation particles, $m$ and $n$ respectively. To determine if the origin of the inaccuracies lies in the multiple activation particles per channel, some simulations were performed with a modified sodium channel incorporating just a single activation particle $m$ rather than the normal three particles. The single $m$-particle statistics were well described by the Fox algorithm in these simulations, confirming that the problem with the Fox SDE formulation is that it does not capture the effect of small numbers of channels flicking briefly open or closed due to the combined action of multiple gating particles per channel.

\section{CONCLUSIONS AND FUTURE WORKS}

\section{A. Conclusions}

The differences between the noise term statistics estimated in this study from the Chow \& White algorithm and the theoretical statistics of the Fox algorithm given by (6) and (7) appear to be sufficient to explain the inaccuracies in AP statistics described by [3] and [4].

\section{B. Future Works}

It remains to derive an analytical expression for the error in the Fox approximation [1], [2] and to determine if an improved SDE formulation can be derived that accurately captures the combined effects of a channel's multiple gating particles on the statistics of the channel's openings and closings.

\section{ACKNOWLEDGMENTS}

The author thanks Faheem Dinath and Melissa Perri for computer programming assistance. This work was supported by the Barber-Gennum Chair Endowment.

\section{REFERENCES}

[1] R. F. Fox, "Stochastic versions of the Hodgkin-Huxley equations," Biophys. J., vol. 72, no. 5, pp. 2068-2074, May 1997.

[2] R. F. Fox and Y.-N. Lu, "Emergent collective behavior in large numbers of globally coupled independently stochastic ion channels," Phys. Rev. E, vol. 49, no. 4, pp. 3421-3431, 1994.

[3] H. Mino, J. T. Rubinstein, and J. A. White, "Comparison of algorithms for the simulation of action potentials with stochastic sodium channels," Ann. Biomed. Eng., vol. 30, no. 4, pp. 578-587, Apr. 2002.

[4] I. C. Bruce, "Implementation issues in approximate methods for stochastic Hodgkin-Huxley models," Ann. Biomed. Eng., vol. 35, no. 2, pp. 315-318, Feb. 2007.

[5] J. A. White, J. T. Rubinstein, and A. R. Kay, "Channel noise in neurons," Trends Neurosci., vol. 23, no. 3, pp. 131-137, Mar. 2000.

[6] J. T. Rubinstein, "Threshold fluctuations in an $N$ sodium channel model of the node of Ranvier," Biophysical Journal, vol. 68, pp. 779785, March 1995.

[7] I. C. Bruce, M. W. White, L. S. Irlicht, S. J. O'Leary, S. Dynes, E. Javel, and G. M. Clark, "A stochastic model of the electrically stimulated auditory nerve: Single-pulse response," IEEE Trans. Biomed. Eng., vol. 46, no. 6, pp. 617-629, June 1999.

[8] I. C. Bruce, L. S. Irlicht, M. W. White, S. J. O'Leary, S. Dynes, E. Javel, and G. M. Clark, "A stochastic model of the electrically stimulated auditory nerve: Pulse-train response," IEEE Trans. Biomed. Eng., vol. 46, no. 6, pp. 630-637, June 1999.

[9] J. T. Rubinstein, B. S. Wilson, C. C. Finley, and P. J. Abbas, "Pseudospontaneous activity: Stochastic independence of auditory nerve fibers with electrical stimulation," Hear. Res., vol. 127, pp. 108-118, 1999.

[10] A. J. Matsuoka, J. T. Rubinstein, P. J. Abbas, and C. A. Miller, "The effects of interpulse interval on stochastic properties of electrical stimulation: Models and measurements," IEEE Trans. Biomed. Eng., vol. 48, no. 4, pp. 416-424, Apr. 2001.

[11] C. L. Runge-Samuelson, P. J. Abbas, J. T. Rubinstein, C. A. . Miller, and B. K. Robinson, "Response of the auditory nerve to sinusoidal electrical stimulation: effects of high-rate pulse trains," Hear. Res., vol. 194, no. 1-2, pp. 1-13, Aug. 2004.

[12] H. Mino, J. T. Rubinstein, C. A. Miller, and P. J. Abbas, "Effects of electrode-to-fiber distance on temporal neural response with electrical stimulation," IEEE Trans. Biomed. Eng., vol. 51, no. 1, pp. 13-20, Jan. 2004.

[13] H. Mino and J. T. Rubinstein, "Effects of neural refractoriness on spatio-temporal variability in spike initiations with electrical stimulation," IEEE Trans. Neural Syst. Rehabil. Eng., vol. 14, no. 3, pp. 273-280, Sept. 2006.

[14] I. C. Bruce, M. W. White, L. S. Irlicht, S. J. O'Leary, and G. M. Clark, "The effects of stochastic neural activity in a model predicting intensity perception with cochlear implants: Low-rate stimulation," IEEE Trans. Biomed. Eng., vol. 46, no. 12, pp. 1393-1404, December 1999.

[15] W. D. Ferguson, L. M. Collins, and D. W. Smith, "Psychophysical threshold variability in cochlear implant subjects," Hear. Res., vol. 180, no. 1-2, pp. 101-113, June 2003.

[16] R. S. Hong and J. T. Rubinstein, "High-rate conditioning pulse trains in cochlear implants: Dynamic range measures with sinusoidal stimuli," J. Acoust. Soc. Am., vol. 114, no. 6 Pt 1, pp. 3327-3342, Dec. 2003.

[17] — - "Conditioning pulse trains in cochlear implants: effects on loudness growth," Otol. Neurotol., vol. 27, no. 1, pp. 50-56, Jan. 2006.

[18] A. Hodgkin and A. Huxley, "A quantitative description of membrane current and its application to conduction and excitation in nerve," $J$. Physiol., vol. 117, pp. 500-44, 1952.

[19] A. F. Strassberg and L. J. DeFelice, "Limitations of the HodgkinHuxley formalism: effects of single channel kinetics on transmembrane voltage dynamics," Neural Comput., vol. 5, no. 6, pp. 843-855, 1993.

[20] C. C. Chow and J. A. White, "Spontaneous action potentials due to channel fluctuations," Biophys. J., vol. 71, pp. 3013-3021, December 1996.

[21] H. C. Tuckwell and P. Lansky, "On the simulation of biological diffusion processes," Comput. Biol. Med., vol. 27, no. 1, pp. 1-7, Jan. 1997. 